\title{
ON HYPERGEOMETRIC SUMMABILITY INVOLVING INFINITE LIMITS
}

\author{
S. K. BASU
}

1. Introduction. The theory of Hausdorff summability has engaged the attention of a number of writers in recent years (see [7, Chap. $\mathrm{XI}]$ ). In connection with the theory of continued fractions Garabedian and Wall $[6, \S 5]$ have defined the hypergeometric summability which, like Cesàro and Hölder summabilities, is a special type of Hausdorff summability. The paper referred to above deals with the case of finite limits, i.e., it considers summability of a sequence summable by a hypergeometric method (H.G. method) to a finite limit. In the present paper we mainly consider some hypergeometric methods which sum a sequence to $\infty$ and compare their total relative strength. ${ }^{1}$

2. The Hausdorff transformation of a sequence ${ }^{2}\left\{s_{n}\right\}$ is defined by

$$
\sigma_{n}=\sum_{k=0}^{n}\left(\begin{array}{l}
n \\
k
\end{array}\right) \Delta^{n-k_{\mu_{k}}} \cdot s_{k} \quad(n=0,1,2, \cdots)
$$

where $\left\{\mu_{n}\right\}$ is a given factor sequence. ${ }^{3}$ In case (1) is a regular transformation, $\left\{\mu_{n}\right\}$ is called a regular moment sequence and has the representation

$$
\mu_{n}=\int_{0}^{1} u^{n} d \chi(u) \quad(n=0,1,2, \cdots),
$$

where $\chi(u)$, termed the regular mass function, is a function of bounded variation in the closed interval $\langle 0,1\rangle{ }^{4}$ If $H$ denotes a regular transformation of the form (1) as defined by (2), we use the symbol $H \sim \chi .^{5}$ The transformation (1), when regular, is said to be totally regular in case $s_{n} \rightarrow \infty$ implies $\sigma_{n} \rightarrow \infty .^{6}$

Received by the editors, March 30, 1953.

${ }^{1}$ For the notion of total relative strength see $[3$, p. 51 , footnote].

2 The numbers with which we are concerned here are all supposed to be real.

${ }^{3}$ For properties of Hausdorff transformation see [7, loc. cit.] or [8].

${ }^{4}$ For properties of regular mass functions see $[5$, p. 193] or $[7$, loc. cit.].

5 This symbol is due to Rogosinski, see [9, p. 167].

${ }^{6}$ For conditions of total regularity of the transformation (1) see [2, Lemma 1]. The necessary and sufficient condition of total regularity of a regular transformation (1), in terms of the mass function, is that $\chi(x) \geqq 0$ and $\uparrow$ in $\langle 0,1\rangle$. (See [2, proof of Lemma 1$]$ or $[6$, p. 186]). Such a mass function will be termed a totally regular mass function. 
In (1) let us take a factor sequence $\left\{\lambda_{n}\right\}$ defined by

(3) $\lambda_{0}=1, \lambda_{n}=\frac{\alpha(\alpha+1) \cdots(\alpha+n-1) \beta(\beta+1) \cdots(\beta+n-1)}{\gamma(\gamma+1) \cdots(\gamma+n-1) \cdot 1 \cdot 2 \cdots \cdot n}$

$$
(n=1,2,3, \cdots) \text {, }
$$

where $\alpha, \beta, \gamma>0$ are given constants. In case $\sigma_{n} \rightarrow l(-\infty \leqq l \leqq \infty)^{7}$ the sequence $\left\{\lambda_{n}\right\}$ defines the hypergeometric summability denoted by $(H, \alpha, \beta, \gamma)$. We shall also use the same symbol for the hypergeometric matrix defined by $\left\{\lambda_{n}\right\}$.

The Cesàro method $C_{\kappa}$ of order $\kappa>-1$ is defined by

$$
\mu_{n}=\frac{1}{\left(\begin{array}{c}
n+\kappa \\
n
\end{array}\right)} \quad(n=0,1,2, \cdots) \text {. }
$$

The theory of H.G. summability becomes more comprehensible when the H.G. matrix is interpreted in the light of its relation to the Cesàro matrices, the connecting link being the formulae:

$$
\begin{array}{rr}
(H, \alpha, \beta, \gamma)=C_{\alpha-1}^{-1} C_{\beta-1}^{-1} C_{\gamma-1} & (\alpha, \beta, \gamma>0), \\
(H, \alpha, \beta, \gamma) \approx C_{\gamma-\alpha-\beta+1} & (\alpha, \beta, \gamma>0, \gamma>\alpha+\beta-2)
\end{array}
$$

$[1$, p. 707], the latter expressing the equivalence of the two methods.

It follows from (5) that $(H, \alpha, \beta, \gamma)$ is regular for $\gamma \geqq \alpha+\beta-1$. As a particular case of (4) we have

$$
(H, 1,1, \gamma+1)=C_{0}^{-1} C_{0}^{-1} C_{\gamma}=C_{\gamma} \quad(\gamma>-1),
$$

since $C_{0}=E$, the identity matrix.

3. The discussion of the question of total regularity of the general H.G. method $(H, \alpha, \beta, \gamma)$ is somewhat complicated and is therefore deferred to $\S 6$. We first consider some simple cases and our results are formulated under (i)-(vi). They (or particular cases thereof) generally correspond to the results given under (5.4) of [6].

Taking $\beta=1$ in (3) we have the method $(H, \alpha, 1, \gamma)$ which is totally regular for $\gamma>\alpha$ as is clear from (4), since $C_{\gamma-1} / C_{\alpha-1}$ is totally regular (see $[7$, p. 101]).

For $\alpha, \beta \geqq 1$, we have

$$
\frac{C_{\gamma}}{(H, \alpha, \beta, \gamma+1)}=C_{\alpha-1} C_{\beta-1} .
$$

${ }^{7}$ Unless otherwise stated $l$ is finite. 
Since $C_{\alpha-1}, C_{\beta-1}$ are regular, so also is $C_{\alpha-1} C_{\beta-1}$ and hence the $(H, \alpha, \beta, \gamma+1)$ summability of a sequence to $l$ implies its $C_{\gamma}$ summability to $l$; in symbol

$$
C_{\gamma} \supset(H, \alpha, \beta, \gamma+1) .
$$

As $C_{\alpha-1}, C_{\beta-1}$ are totally regular, the above result is true also when $l=\infty$ (see [7, proof of Theorem 210, p. 263]; in symbol

$$
C_{\gamma} \text { t.s. }(H, \alpha, \beta, \gamma+1) \quad(\alpha, \beta \geqq 1, \gamma>-1)
$$

(t.s. means totally stronger than). ${ }^{8}$ Similarly, it can be easily shown that

$$
(H, \alpha, \beta, \gamma+1) \text { t.s. } C_{\gamma}
$$$$
(0<\alpha, \beta \leqq 1, \gamma>-1) \text {. }
$$

Since $C_{\gamma}$ is totally regular for $\gamma>0$, it follows from (ii) that $(H, \alpha, \beta, \gamma+1)$ is totally regular for $0<\alpha, \beta \leqq 1$ and $\gamma>0 .{ }^{9}$

4. We shall require the following lemma.

Leмma 1. Of two regular Hausdorff methods with nonvanishing moment sequences, each cannot be totally stronger than the other unless they are identical [2, Lemma 2].

For $\alpha>0$,

$$
(H, \alpha, 1, \alpha+1) \approx C_{1} .
$$

We enquire about the total relative strength of the two matrices in (6). For the regular matrix $(H, \alpha, 1, \alpha+1) / C_{1}$ the factor sequence is

$$
\mu_{n}=\alpha \cdot \frac{n+1}{n+\alpha}=\alpha\left\{1+\frac{1-\alpha}{n+\alpha}\right\} .
$$

The sequence $\{1 /(n+\alpha)\}$ is obviously totally monotone for $\alpha>0$. Hence for $0<\alpha<1,\left\{\mu_{n}\right\}$ is also totally monotone. Accordingly,

$$
(H, \alpha, 1, \alpha+1) \text { t.s. } C_{1}
$$$$
(0<\alpha<1) \text {; }
$$

and therefore by Lemma 1 ,

$$
C_{1} \text { n.t.s. }(H, \alpha, 1, \alpha+1) \text {. }
$$

Similarly,

$$
C_{1} \text { t.s. }(H, \alpha, 1, \alpha+1)
$$$$
(\alpha>1)
$$

${ }^{8}$ In the sequel we shall use the notation $A$ n.t.s. $B$ to denote the fact that $A$ and $B$ being two methods of summability, $A \supset B$, but it is not true that $A$ t.s. $B$.

${ }^{9}$ See Theorem 4 (i) below of which this is a particular case. 
and hence by Lemma 1 ,

$$
(H, \alpha, 1, \alpha+1) \text { n.t.s. } C_{1} .
$$

We have

$$
\begin{gathered}
T=\frac{\left(H, \alpha, \beta, \gamma^{\prime}\right)}{\left(H, \alpha^{\prime}, \beta^{\prime}, \gamma\right)}=\frac{C_{\gamma^{\prime}-1}}{C_{\gamma-1}} \cdot \frac{C_{\alpha^{\prime}-1}}{C_{\alpha-1}} \cdot \frac{C_{\beta^{\prime}-1}}{C_{\beta-1}} \\
\left(\alpha, \beta, \gamma>0, \alpha^{\prime} \geqq \alpha, \beta^{\prime} \geqq \beta, \gamma^{\prime} \geqq \gamma, \gamma>\alpha+\beta-1, \gamma^{\prime}>\alpha^{\prime}+\beta^{\prime}-1\right),
\end{gathered}
$$

so that $T$ is a totally regular matrix. Hence

$$
\left(H, \alpha, \beta, \gamma^{\prime}\right) \text { t.s. }\left(H, \alpha^{\prime}, \beta^{\prime}, \gamma\right) .
$$

Again, we have

$$
(H, \alpha, 1, \alpha+1) \approx(H, \beta, 1, \beta+1) \quad(\alpha, \beta>0) .
$$

Regarding the total relative strength of these two matrices we easily find that

$$
(H, \alpha, 1, \alpha+1) \text { t.s. }(H, \beta, 1, \beta+1) \quad(\beta>\alpha>0) .
$$

Consequently,

$$
(H, \beta, 1, \beta+1) \text { n.t.s. }(H, \alpha, 1, \alpha+1) .
$$

(vi) may be written in the equivalent form

$$
\frac{C_{\alpha}}{C_{\alpha-1}} \text { t.s. } \frac{C_{\beta}}{C_{\beta-1}} \quad(\beta>\alpha>0),
$$

so that we may say that the matrix $C_{\alpha} / C_{\alpha-1}$ loses its total strength with the increase of $\alpha$.

5. The important equivalence relation

$$
C_{\alpha} C_{\beta} \approx C_{\alpha+\beta} \quad(\alpha, \beta, \alpha+\beta>-1)
$$

has been studied by various writers in connection with the Hausdorff summability. ${ }^{10} \mathrm{We}$ would like to study the total relative strength of the two matrices $C_{\alpha} C_{\beta}$ and $C_{\alpha+\beta}$. The following theorems will be proved.

Theorem 1. For $\alpha, \beta, \alpha+\beta>-1$,

$$
C_{\alpha} C_{\beta} \text { t.s. } C_{\alpha+\beta}
$$

and

${ }^{10}$ See e.g. $[8$, p. $90 ; 7$, pp. 118,$265 ; 1$, p. 706$]$. 


$$
C_{\alpha+\beta} \text { t.s. } C_{\alpha} C_{\beta}
$$

and

$$
C_{\alpha} C_{\beta} \text { n.t.s. } C_{\alpha+\beta}
$$

when $\alpha \beta<0$.

We require the following lemma.

Lemma 2. For $\alpha, \beta, \alpha+\beta>-1, t>0$,

(i) $(n+t+1)^{-k}-(n+t+\alpha+1)^{-k}>k \alpha(n+t+\alpha+1)^{-k-1}$,

(ii) $(n+t+\beta+1)^{-k}-(n+t+\alpha+\beta+1)^{-k}$

$$
\begin{aligned}
<k \alpha(n+t+\beta+1)^{-k-1} & \\
& (n=0,1,2, \cdots ; k=1,2,3, \cdots) .
\end{aligned}
$$

Proof of Lemma. In the well known inequality

$$
r x^{r-1}(x-y)>x^{r}-y^{r}>r y^{r-1}(x-y),
$$

where $x, y>0, x \neq y$, and $r<0$, we put

$$
x=n+t+1, \quad y=n+t+\alpha+1 \quad \text { and } r=-k \text {. }
$$

Then the latter part gives (i).

Again, putting

$$
x=n+t+\beta+1, \quad y=n+t+\alpha+\beta+1, \quad \text { and } r=-k
$$

in the first part of (8), we obtain (ii).

Proof of Theorem 1. From (7),

$$
C_{\alpha} C_{\beta} / C_{\alpha+\beta} \approx E \text {. }
$$

To prove (i) we have to show that for $\alpha, \beta, \alpha+\beta>-1$ and $\alpha \beta>0$, the matrix $C_{\alpha} C_{\beta} / C_{\alpha+\beta}$ is totally regular. If $\left\{\mu_{n}\right\}$ be the moment sequence for this regular matrix, then

$$
\begin{aligned}
\mu_{n} & =\frac{\left(\begin{array}{c}
n+\alpha+\beta \\
n
\end{array}\right)}{\left(\begin{array}{c}
n+\alpha \\
n
\end{array}\right)\left(\begin{array}{c}
n+\beta \\
n
\end{array}\right)} \\
& =\frac{\Gamma(\alpha+1) \Gamma(\beta+1)}{\Gamma(\alpha+\beta+1)} \cdot \frac{\Gamma(n+1) \Gamma(n+\alpha+\beta+1)}{\Gamma(n+\alpha+1) \Gamma(n+\beta+1)} .
\end{aligned}
$$

The corresponding moment function ${ }^{11} \mu(t)$ is then given by

${ }^{11}$ For the definition and properties of moment functions see [8, pp. 95, 102-103]. 


$$
\mu(t)=\frac{\Gamma(\alpha+1) \Gamma(\beta+1)}{\Gamma(\alpha+\beta+1)} \cdot \frac{\Gamma(t+1) \Gamma(t+\alpha+\beta+1)}{\Gamma(t+\alpha+1) \Gamma(t+\beta+1)} \quad(t \geqq 0) .
$$

Put

$$
\mu(t)=e^{\rho(t)} .
$$

Then for $t>0$,

$$
\begin{aligned}
\rho^{\prime}(t)= & \frac{\Gamma^{\prime}(t+1)}{\Gamma(t+1)}+\frac{\Gamma^{\prime}(t+\alpha+\beta+1)}{\Gamma(t+\alpha+\beta+1)}-\frac{\Gamma^{\prime}(t+\alpha+1)}{\Gamma(t+\alpha+1)} \\
& -\frac{\Gamma^{\prime}(t+\beta+1)}{\Gamma(t+\beta+1)} \\
= & \sum_{n=0}^{\infty}\left[\frac{1}{n+t+\alpha+1}+\frac{1}{n+t+\beta+1}-\frac{1}{n+t+1}\right.
\end{aligned}
$$

$$
\left.-\frac{1}{n+t+\alpha+\beta+1}\right]
$$

(see, e.g., $[10$, p. 149]) and hence for $k=1,2,3, \cdots$,

$$
\begin{aligned}
\frac{(-1)^{k} \rho^{(k)}(t)}{(k-1) !}= & \sum_{n=0}^{\infty}\left[(n+t+1)^{-k}+(n+t+\alpha+\beta+1)^{-k}\right. \\
& \left.-(n+t+\alpha+1)^{-k}-(n+t+\beta+1)^{-k}\right] \\
= & \sum_{n=0}^{\infty}\left[\left\{(n+t+1)^{-k}-(n+t+\alpha+1)^{-k}\right\}\right. \\
& \left.-\left\{(n+t+\beta+1)^{-k}-(n+t+\alpha+\beta+1)^{-k}\right\}\right] \\
& (t>0),
\end{aligned}
$$

the term-by-term differentiation being justified by the uniform convergence of the differentiated series. Hence as $\alpha, \beta, \alpha+\beta>-1$, with the help of Lemma 2, we have

$$
\begin{aligned}
& \frac{(-1)^{k} \rho^{(k)}(t)}{(k-1) !}>k \alpha \sum_{n=0}^{\infty}\left[(n+t+\alpha+1)^{-k-1}\right. \\
&\left.-(n+t+\beta+1)^{-k-1}\right] \quad(t>0) .
\end{aligned}
$$

When $\alpha \beta>0$ either $\alpha, \beta>0$ or $-1<\alpha, \beta<0$.

Case I. $\alpha, \beta>0$. Suppose $0<\alpha \leqq \beta$. Then from (10),

$$
(-1)^{k} \rho^{(k)}(t)>0 \quad(t>0 ; k=1,2,3, \cdots),
$$

and as $\rho(0)=\rho(+0)$, when $0<\alpha \leqq \beta, \mu(t)$ is totally monotone for $t \geqq 0$ 
(see [8, p. 95]). It follows from symmetry that for $0<\beta<\alpha$ also $\mu(t)$ is totally monotone.

Hence $C_{\alpha} C_{\beta} / C_{\alpha+\beta}$ is totally regular as it is regular and therefore $C_{\alpha+\beta} / C_{\alpha} C_{\beta}$ is not totally regular by Lemma 1 .

Case II. $-1<\alpha, \beta<0$. When $-1<\beta \leqq \alpha<0, \alpha+\beta>-1$ from (10) for $t>0$,

$$
\begin{aligned}
\frac{(-1)^{k} \rho^{(k)}(t)}{(k-1) !}> & -k \alpha \sum_{n=0}^{\infty}\left[(n+t+\beta+1)^{-k-1}\right. \\
& \left.-(n+t+\alpha+1)^{-k-1}\right]>0
\end{aligned}
$$

and as before we find that for $-1<\alpha, \beta<0, \alpha+\beta>-1, C_{\alpha} C_{\beta} / C_{\alpha+\beta}$ is totally regular but not $C_{\alpha+\beta} / C_{\alpha} C_{\beta}$. (i) now follows.

To prove (ii) we have to consider the moment function $\nu(t)=\frac{\Gamma(\alpha+\beta+1)}{\Gamma(\alpha+1) \Gamma(\beta+1)} \cdot \frac{\Gamma(t+\alpha+1) \Gamma(t+\beta+1)}{\Gamma(t+1) \Gamma(t+\alpha+\beta+1)}=e^{\tau(t)}$, say $(t \geqq 0)$.

Suppose $\alpha \beta<0$, so that either $\alpha$ or $\beta$ is negative. There will be two different cases according as $\alpha+\beta \geqq 0$ or $<0$.

Case I. Suppose $-1<\beta<0<\alpha,-1<\alpha+\beta<0$. Proceeding as in (i) we have now, for $t>0$,

$$
\begin{aligned}
\frac{(-1)^{k} \tau^{(k)}(t)}{(k-1) !}= & \sum_{n=0}^{\infty}\left[\left\{(n+t+\beta+1)^{-k}-(n+t+\alpha+\beta+1)^{-k}\right\}\right. \\
& \left.-\left\{(n+t+1)^{-k}-(n+t+\alpha+1)^{-k}\right\}\right] \\
> & k \alpha \sum_{n=0}^{\infty}\left[(n+t+\alpha+\beta+1)^{-k-1}-(n+t+1)^{-k-1}\right] \\
> & 0 .
\end{aligned}
$$

Also, from symmetry it follows that for

$$
\begin{gathered}
-1<\alpha<0<\beta, \quad-1<\alpha+\beta<0, \\
(-1)^{k} \tau^{(k)}(t)>0 .
\end{gathered}
$$

Case II. Suppose $-1<\beta<0<\alpha, \alpha+\beta \geqq 0$. In this case we shall have for $t>0$,

$$
\begin{aligned}
\frac{(-1)^{k} \tau^{(k)}(t)}{(k-1) !} & >-k \beta \sum_{n=0}^{\infty}\left[(n+t+1)^{-k-1}-(n+t+\alpha+\beta+1)^{-k-1}\right] \\
& >0 .
\end{aligned}
$$

Hence in either case 


$$
(-1)^{k} \tau^{(k)}(t)>0
$$

and as before, $C_{\alpha+\beta} / C_{\alpha} C_{\beta}$ is totally regular for

$$
\alpha, \beta, \alpha+\beta>-1 \text { and } \alpha \beta<0 .
$$

Consequently, $C_{\alpha} C_{\beta} / C_{\alpha+\beta}$ is not totally regular. This proves (ii).

From (9) we have, in terms of the H.G. matrix,

$$
(H, \alpha+1, \beta+1, \alpha+\beta+1) \approx E .
$$

It then follows from Theorem 1 that for $\alpha, \beta, \alpha+\beta>-1,(H, \alpha+1$, $\beta+1, \alpha+\beta+1)$ is totally regular when $\alpha \beta<0$ and not totally regular when $\alpha \beta>0$.

6. Total regularity of $(H, \alpha, \beta, \gamma)$. We have already considered some particular cases of $(H, \alpha, \beta, \gamma)$ for total regularity. We now turn to the general case. It may be noted, first of all, that the $(H, \alpha, \beta, \gamma)$ method, though regular, is not always totally regular for $\gamma \geqq \alpha+\beta-1$, as is clear from the following theorem.

THEOREM 2. The regular method $(H, \alpha, \beta, \gamma)$ is not totally regular when

$$
\alpha+\beta-1 \leqq \gamma \leqq \alpha \beta \quad(\alpha, \beta, \gamma>0){ }^{12}
$$

Proof. We have

$$
\Delta \lambda_{0}=\lambda_{0}-\lambda_{1}=1-\frac{\alpha \beta}{\gamma}<0 \quad \text { when } \gamma<\alpha \beta .
$$

Hence $\left\{\lambda_{n}\right\}$ is not totally monotone and as such $(H, \alpha, \beta, \gamma)$ cannot be totally regular for $\alpha+\beta-1<\gamma<\alpha \beta$.

Again, for $\gamma=\alpha \beta$,

$$
\begin{aligned}
\Delta^{2} \lambda_{0} & =\lambda_{0}-2 \lambda_{1}+\lambda_{2} \\
& =1-\frac{2 \alpha \beta}{\gamma}+\frac{\alpha(\alpha+1) \beta(\beta+1)}{\gamma(\gamma+1) \cdot 1 \cdot 2} \\
& =\frac{(\alpha+1)(\beta+1)}{2(\gamma+1)}-1 .
\end{aligned}
$$

Hence $\Delta^{2} \lambda_{0}<0$ as $\gamma>\alpha+\beta-1$.

Therefore, for $\alpha+\beta-1<\gamma=\alpha \beta,(H, \alpha, \beta, \gamma)$ is not totally regular. Lastly, writing $\alpha-1$ and $\beta-1$ for $\alpha$ and $\beta$ respectively in the

12 The case when $\alpha$ or $\beta=1$ is trivial for our purpose; for, in such a case the total regularity or otherwise of $(H, \alpha, \beta, \gamma)$ can at once be detected by inspection. In what follows, we shall suppose that $\alpha, \beta \neq 1$. 
second part of Theorem 1(i) we find that when $\alpha, \beta, \alpha+\beta-1>0$, $(H, \alpha, \beta, \gamma)$ is not totally regular for $\gamma=\alpha+\beta-1<\alpha \beta$. This completes the proof of the theorem.

We note that the result obtained in the above theorem for $\gamma=\alpha \beta$ $>\alpha+\beta-1$, when interpreted in terms of the Cesàro matrices, shows that $C_{\alpha \beta-1} / C_{\alpha-1} C_{\beta-1}$ is not totally regular for $(\alpha-1)(\beta-1)>0$ $(\alpha, \beta>0)$.

It follows from Theorem 2 that a necessary condition for total regularity of a regular method $(H, \alpha, \beta, \gamma)$ is that

$$
\gamma \geqq \alpha+\beta-1, \alpha \beta \quad(\alpha, \beta, \gamma>0) .
$$

It is natural to enquire if this condition is sufficient as well. The answer to this question, which is, of course, not complete, is contained in the following two theorems.

TheOREM 3. For $\gamma \geqq \alpha+\beta-1>\alpha \beta(\alpha, \beta>0)$, the method $(H, \alpha, \beta, \gamma)$ is totally regular.

Theorem 4. For $\gamma>\alpha \beta>\alpha+\beta-1,{ }^{13}$

(i) $(H, \alpha, \beta, \gamma)$ is totally regular when $0<\alpha, \beta<1$ and $\gamma>\alpha$ or $\beta .{ }^{14}$

(ii) $(H, \alpha, \beta, \gamma)$ is not totally regular when $\alpha, \beta>1$.

Proof of Theorem 3. We have seen in $\S 5$ that $(H, \alpha, \beta, \alpha+\beta-1)$ is totally regular when $(\alpha-1)(\beta-1)<0$, i.e. for $\alpha+\beta-1>\alpha \beta$ $(\alpha, \beta>0)$. Since by $(\mathrm{v})$, for $\gamma \geqq \alpha+\beta-1$,

$$
(H, \alpha, \beta, \gamma) \text { t.s. }(H, \alpha, \beta, \alpha+\beta-1) \text {, }
$$

it follows that $(H, \alpha, \beta, \gamma)$ is totally regular for $\gamma \geqq \alpha+\beta-1>\alpha \beta$ $(\alpha, \beta>0)$.

For the proof of the last theorem we require two more lemmas.

Lemma 3. Let $H_{r} \sim \phi_{r}(r=1,2,3)$ be three regular Hausdorff matrices such that

$$
H_{3}=H_{1} H_{2}
$$

Then $^{15}$

$$
\phi_{3}(x)=\phi_{2}(x)+\int_{x}^{1} \phi_{1}\left(\frac{x}{t}\right) d \phi_{2}(t) \quad(0<x<1),
$$

the integral being taken in the Lebesgue-Stieltjes sense.

${ }^{13}$ The case for $\gamma=\alpha \beta>\alpha+\beta-1$ has already been considered.

${ }^{14}$ We restrict ourselves here to the case $\gamma>\alpha$ or $\beta$. Whether $(H, \alpha, \beta, \gamma)$ is totally regular whenever $\gamma>\alpha \beta>\alpha+\beta-1$, and $0<\alpha, \beta<1$, still remains an open question.

${ }^{15}$ See [5, pp. 196, 201]; also cf. [4, p. 504]. 
Lemma 4. For $\alpha, \beta>1$, let $B=C_{\alpha-1} C_{\beta-1} \sim \phi$. Then

$$
\phi(x)=1-(\beta-1) \int_{x}^{1}\left(1-\frac{x}{t}\right)^{\alpha-1}(1-t)^{\beta-2} d t \quad(0<x<1) .
$$

Proof. Let

$$
C_{p-1} \sim \phi_{p} \quad(p=\alpha, \beta>1)
$$

so that

$$
\phi_{p}(x)=1-(1-x)^{p-1} \quad(0 \leqq x \leqq 1) .
$$

Since $B=C_{\alpha-1} C_{\beta-1}$, by Lemma 3,

$$
\begin{array}{rlrl}
\phi(x) & =\phi_{\beta}(x)+\int_{x}^{1} \phi_{\alpha}\left(\frac{x}{t}\right) d \phi_{\beta}(t) & (0<x<1) \\
=1-(1-x)^{\beta-1}+(\beta-1) \int_{x}^{1}\left[1-\left(1-\frac{x}{t}\right)^{\alpha-1}\right] & (1-t)^{\beta-2} d t ;
\end{array}
$$

whence

$$
\phi(x)=1-(\beta-1) \int_{x}^{1}\left(1-\frac{x}{t}\right)^{\alpha-1}(1-t)^{\beta-2} d t .
$$

Proof of Theorem 4. (i) When $\alpha \beta>\alpha+\beta-1$, either $0<\alpha, \beta<1$ or $\alpha, \beta>1$. By hypothesis, $0<\alpha, \beta<1$; and suppose $\gamma>\alpha$, so that the condition $\gamma>\alpha \beta$ is not violated. Now

$$
(H, \alpha, \beta, \gamma)=\frac{C_{\gamma-1}}{C_{\alpha-1} C_{\beta-1}}=\frac{C_{\gamma-1}}{C_{\alpha-1}} \cdot \frac{1}{C_{\beta-1}} .
$$

Since $C_{\gamma-1} / C_{\alpha-1}$ and $1 / C_{\beta-1}$ are both totally regular and since $(H, \alpha, \beta, \gamma)$ is symmetrical in $\alpha, \beta$ the result at once follows.

(ii) First suppose $1<\alpha, \beta<2$. Suppose, if possible, that $(H, \alpha, \beta, \gamma)$ is totally regular for $\gamma>\alpha \beta$. Writing $A$ for $(H, \alpha, \beta, \gamma)$ and $B$ for $C_{\alpha-1} C_{\beta-1} \sim \phi$ which is now totally regular, we have

$$
A=\frac{C_{\gamma-1}}{C_{\alpha-1} C_{\beta-1}}=\frac{C_{\gamma-1}}{B}
$$

a totally regular matrix. Hence if $A \sim \psi$ and $C_{\gamma-1} \sim \phi_{\gamma}$, by Lemma 3,

$$
\phi_{\gamma}(x)=\phi(x)+\int_{x}^{1} \psi\left(\frac{x}{t}\right) d \phi(t)
$$

so that 


$$
\int_{x}^{1} \psi\left(\frac{x}{t}\right) d \phi(t)=\phi_{\gamma}(x)-\phi(x)=f(x), \text { say. }
$$

By Lemma 4,

$$
\begin{aligned}
f(x)=1 & -(1-x)^{\gamma-1} \\
- & {\left[1-(\beta-1) \int_{x}^{1}\left(1-\frac{x}{t}\right)^{\alpha-1}(1-t)^{\beta-2} d t\right], }
\end{aligned}
$$

that is,

$$
\begin{aligned}
f(x)=(\beta-1) \int_{x}^{1}\left(1-\frac{x}{t}\right)^{\alpha-1}(1-t)^{\beta-2} d t- & (1-x)^{\gamma-1} \\
& (0<x<1) .
\end{aligned}
$$

Since, for $0<x<t \leqq 1$,

$$
1-\frac{x}{t}>0
$$

and $0<\alpha-1<1$, by a well known inequality

$$
\left(1-\frac{x}{t}\right)^{\alpha-1}<1-(\alpha-1) \frac{x}{t} .
$$

Therefore,

$$
\int_{x}^{1}\left(1-\frac{x}{t}\right)^{\alpha-1}(1-t)^{\beta-2} d t<\int_{x}^{1}\left[1-(\alpha-1) \frac{x}{t}\right](1-t)^{\beta-2} d t,
$$

that is,

$$
\begin{aligned}
\int_{x}^{1}\left(1-\frac{x}{t}\right)^{\alpha-1}(1 & -t)^{\beta-2} d t \\
& <\frac{(1-x)^{\beta-1}}{\beta-1}-(\alpha-1) x \int_{x}^{1} \frac{1}{t}(1-t)^{\beta-2} d t .
\end{aligned}
$$

Again, for $0<\alpha<1$ and $1<\beta<2$,

$$
\frac{1}{t}(1-t)^{\beta-2}>\frac{1}{t}(1-t) .
$$

Hence

$$
\int_{x}^{1} \frac{1}{t}(1-t)^{\beta-2} d t>\int_{x}^{1} \frac{1}{t}(1-t) d t,
$$


or

$$
-\int_{x}^{1} \frac{1}{t}(1-t)^{\beta-2} d t<-\int_{x}^{1} \frac{1}{t}(1-t) d t=\log x+1-x .
$$

Consequently, from (13),

$$
\begin{aligned}
\int_{x}^{1}\left(1-\frac{x}{t}\right)^{\alpha-1}(1-t)^{\beta-2} d t & \\
< & <\frac{(1-x)^{\beta-1}}{\beta-1}+(\alpha-1) x[\log x+1-x] .
\end{aligned}
$$

Therefore, from (12),

$$
\begin{aligned}
f(x)< & (1-x)^{\beta-1}-(1-x)^{\gamma-1} \\
& +(\alpha-1)(\beta-1) x[\log x+1-x]=F(x), \\
& \text { say, } \\
& (0<x<1) .
\end{aligned}
$$

Now for the function $F(x)$,

$$
\begin{aligned}
F^{\prime}(x)= & -(\beta-1)(1-x)^{\beta-2}+(\gamma-1)(1-x)^{\gamma-2} \\
& +(\alpha-1)(\beta-1)[\log x+1]+(\alpha-1)(\beta-1)(1-2 x) .
\end{aligned}
$$

Since $F^{\prime}(x) \rightarrow-\infty$ as $x \rightarrow+0$, it follows that

$$
F^{\prime}(x)<0
$$

in an open interval $(0, \epsilon)$ for some $\epsilon>0$. Hence $F(x) \downarrow$ in $(0, \epsilon)$; and as $F(+0)=0$, we must have

$$
F(x)<0 \text { in }\left(0, \epsilon^{\prime}\right) \text { for some } \epsilon^{\prime}>0 .
$$

Hence by (14), $f(x)<0$ in $\left(0, \epsilon^{\prime}\right)$.

Now $\psi(x)$ and $\phi(x)$ being totally regular mass functions,

$$
\int_{x}^{1} \psi\left(\frac{x}{t}\right) d \phi(t) \geqq 0
$$

and therefore, by (11),

$$
f(x) \geqq 0 .
$$

We have thus a contradiction which proves that $(H, \alpha, \beta, \gamma)$ is not totally regular for $1<\alpha, \beta<2$ and $\gamma>\alpha \beta$.

Next suppose $\alpha, \beta>1$ and suppose that $\alpha^{\prime}$ and $\beta^{\prime}$ are two parameters such that 


$$
1<\alpha^{\prime}, \beta^{\prime}<2 \text { and } \alpha \geqq \alpha^{\prime}, \beta \geqq \beta^{\prime} .
$$

Suppose, if possible, that $(H, \alpha, \beta, \gamma)$ is totally regular for $\alpha, \beta>1$ and $\gamma>\alpha \beta$ (and consequently $\gamma>\alpha \beta>\alpha+\beta-1$ ). Then since

$$
\left(H, \alpha^{\prime}, \beta^{\prime}, \gamma\right) \text { t.s. }(H, \alpha, \beta, \gamma) \text {, }
$$

it follows that $\left(H, \alpha^{\prime}, \beta^{\prime}, \gamma\right)$ is totally regular for $1<\alpha^{\prime}, \beta^{\prime}<2$ and $\gamma>\alpha^{\prime} \beta^{\prime}>\alpha^{\prime}+\beta^{\prime}-1$, which is impossible. The proof of (ii) is now complete.

\section{REFERENCES}

1. R. P. Agnew, On methods of summability and mass functions determined by hypergeometric coefficients, Amer. J. Math. vol. 63 (1941) pp. 705-708.

2. S. K. Basu, On the total relative strength of the Hölder and Cesaro methods, Proc. London Math. Soc. (2) vol. 50 (1949) pp. 447-462.

3. - On the total relative strength of the Riesz and Cesdro methods, J. London Math. Soc. vol. 24 (1949) pp. 51-59.

4. H. L. Garabedian, Hausdorff integral transformations, Ann. of Math. vol. 43 (1942) pp. 501-509.

5. H. L. Garabedian, E. Hille, and H. S. Wall, Formulations of the Hausdorff inclusion problem, Duke Math. J. vol. 8 (1941) pp. 193-213.

6. H. L. Garabedian and H. S. Wall, Hausdorff methods of summation and continued fractions, Trans. Amer. Math. Soc. vol. 48 (1940) pp. 185-207.

7. G. H. Hardy, Divergent series, 1949, Oxford University Press.

8. F. Hausdorff, Summationsmethoden und Momentfolgen I, Math. Zeit. vol. 9 (1921) pp. 74-109.

9. W. W. Rogosinski, On Hausdorff methods of summability, Proc. Cambridge Philos. Soc. vol. 38 (1942) pp. 166-192.

10. E. C. Titchmarsh, The theory of functions, 2d ed., 1939, Oxford University Press.

Presidency College, Calcutta 